\title{
Researches on Mathematical Relationship of Five Elements of Containing Notes and Fibonacci Sequence Modulo 5
}

\author{
Zhaoxue Chen \\ School of Medical Instrument \& Food Engineering, University of Shanghai for Science and Technology, Shanghai 200093, China \\ Correspondence should be addressed to Zhaoxue Chen; chenzhaoxue@163.com
}

Received 27 September 2014; Revised 8 December 2014; Accepted 10 December 2014

Academic Editor: Zhaohui Liang

Copyright ( 2015 Zhaoxue Chen. This is an open access article distributed under the Creative Commons Attribution License, which permits unrestricted use, distribution, and reproduction in any medium, provided the original work is properly cited.

\begin{abstract}
Considering the five periods and six qi's theory in TCM almost shares a common basis of stem-branch system with the five elements of containing notes, studying the principle or mathematical structure behind the five elements of containing notes can surely bring a novel view for the five periods and six qi's researches. By analyzing typical mathematical rules included in He tu, Luo shu, and stem-branch theory in TCM as well as the Fibonacci sequence especially widely existent in the biological world, novel researches are performed on mathematical relationship between the five elements of containing notes and the Fibonacci sequence modulo 5. Enlightened by elementary Yin or Yang number grouping principle of $\mathrm{He}$ tu, Luo shu, the 12534 and 31542 key number series of Fibonacci sequence modulo 5 are obtained. And three new arrangements about the five elements of containing notes are then introduced, which have shown close relationship with the two obtained key subsequences of the Fibonacci sequence modulo 5. The novel discovery is quite helpful to recover the scientific secret of the five periods and six qi's theory in TCM as well as that of whole traditional Chinese culture system, but more data is needed to elucidate the TCM theory further.
\end{abstract}

\section{Introduction}

Combining the 10 celestial stems and 12 earth branches, the Chinese sexagenary cycle can be formed, which is widely used in Chinese ancient culture as the most important means of counting time of years, months, or dates. In TCM (Traditional Chinese Medicine), given that each year is associated with a different combination of stems and branches over a cycle of sixty years, all possible climatic constellations of the year may sequentially occur in a specific way, which can be characterized by the stem-branch combination closely related with the five periods and six qi. In other words, the doctrine of the five periods and six qi explains relationships ancient Chinese observers assumed to exist between climate and a broad range of natural phenomena, including human health and illness. And the concepts of the five periods and of the six qi were introduced to distinguish among and specify climatic characteristics of well-defined time periods. By drawing on notions of a cyclical recurrence of calendric terms and by adopting the doctrines of yin yang and of the five elements, an attempt was made to order what may at first glance appear to be disorder, namely, the occurrence of rain and wind, dryness, cold, and heat in the course of the four seasons and over the years. Knowledge of a distinct regularity uncovered in frequent climatic changes not only permitted an understanding of the generation, growth, maturity, and death of numerous phenomena in nature in general but also, more importantly, enabled man to integrate himself into eternal laws governing all existence. The doctrine of the five periods and six qi is outlined in the Su wen in seven "comprehensive discourses," which comprises about one-third of the entire text of the Su wen. But the origin of the notions is unclear and no parallel literary sources outside the Su wen are known that could be used to date the early development of these thoughts [1]. All the same, there are also some important clues in other fields of ancient Chinese culture which can be used as a reference for researchers of the five periods and six qi in TCM. Therein, theory of the five elements of containing notes originated at least before Qin Dynasty is the most notable one, where each combination of a stem and a branch is also vital for description of yin yang and five elemental characteristics and each attributed to a corresponding element named as the so-called 
five elements of containing notes $[2,3]$. The na yin wu xing, that is, the element representing the stem-branch of one's birth-year, is often used to judge one's fate by folk fate calculators up to nowadays and in the Book of the Master Who Embraces Simplicity by Ge Hong (who is a famous Taoist priest in Jin Dynasty) it is recorded that the fate corresponding to the five elements of containing notes decided by the stem-branch combination corresponding to one's birth year can be used as a guidance of color selecting of the medicine to be taken; Ge Hong says in volume number 11 of his inner book of the Master Who Embraces Simplicity: “...According to the book of Yu ce ji and the book of Kai ming jing,..., if one's fate is soil, he is not fit to take medicine with cyan color; medicines with red color are not fit for persons of metal fate; white color is not fit for wood fate; yellow color is not fit for water fate and black color is not fit for fire fate. That is exactly because, according to meaning of the five elements, the wood restricts the soil, the soil restricts the water, the water restricts the fire, the fire restricts the metal and the metal restrict the wood...". Although some of Ge Hong's ideas are contentious with a mysterious tendency of a common Taoist, it is true that his Handbook of Prescriptions for Emergencies inspired the modern discovery of artemisinin [4]. No matter in theory of the TCM five periods and six qi or in theory of the five elements of containing notes, the same 60 cyclical stembranch combinations by years are paid much attention and related judgments or doctrines are formed based on the stem and branch related theory of yin yang and five elements. Considering the five periods and six qi's theory of TCM almost shares a common basis of stem-branch system $[1,2]$, studying the principle or mathematical structure behind the five elements of containing notes can surely bring a novel view for the five periods and six qi's related researches in TCM.

The Fibonacci sequence, that is, $1,1,2,3,5,8,13,21,34,55$, $89,144,233, \ldots$, is a famous series universally used in various modern disciplines such as computer science, optimizing theory, biological mathematics and physics, number theory and combinatorics, and material science [5-9]. Even there is a special formal periodical (namely, The Fibonacci Quarterly) dedicated to Fibonacci sequence related topics and researches [10]. In particular, having close relationship with the Golden Section Number, it is an interesting mathematical sequence that widely exists in the biological world. For example, there is a peculiar pattern in the flower petals of nearly all the flowers; the number of their petals is one number of the Fibonacci sequence. Small flowers of a sunflower, the heart of chrysanthemum, squama on surface of pinecones, and tumors-like structure of pineapple all have shown similar two nearperfect spirals in two opposite spiral directions, respectively. And the ratio of two spiral numbers has close relationship with the Fibonacci sequence. In the pinecones the ratio is $5: 8$, the pineapple is $8: 13$, Marguerite daisy is $21: 34$, and the sunflower is $34: 55, \ldots$; the series of number couples are all from two adjacent numbers of the Fibonacci series exactly. Any face plate of the Asteraceae family has the same characteristics with the sunflower. In animal cells, hollow cores of microtubules constituted by protein polymer, which form the cell cytoskeleton, help to maintain a certain shape and act as "nervous system" of cells. Typical mammalian cell microtubule is constituted by 13 original fibers, of which 5 are dextrorotation fibers and 8 are laevorotation ones (herein, 5, 8, and 13 are all adjacent Fibonacci numbers). Moreover, people have occasionally found a double-microtubule with an outer layer, and it is constituted by 21 original fibers, which happens to be the next number in Fibonacci series. Moreover, it is well known that molecules of the B-DNA expose a double-helix geometric structure, and the helix length of the double-helix DNA structure is 34 angstroms and its radius is 21 angstroms. 34 and 21 happen to be the two adjacent numbers in the Fibonacci sequence [6].

Moreover, the Fibonacci sequence is also found to have close relationship with traditional Chinese culture. Elizabeth Moran and her coauthors have discovered that four of feng shui's (feng shui is another mysterious system in traditional Chinese culture system with almost the same theory basis as TCM) fundamental principles correspond to numbers in the Fibonacci sequence: Taiji (1), yin and yang (2), heaven, earth, and human qi (3), five phases (5), and eight trigrams (8) [11]. And according to some other researchers, the Fibonacci numbers also have close relationship with the most famous two key diagrams of $\mathrm{He}$ tu and Luo shu in ancient Chinese culture $[12,13]$.

In this paper, according to some typical mathematical rules included in $\mathrm{He}$ tu, Luo shu in TCM, novel associations are discovered between the five elements of containing notes and the Fibonacci modular sequence, which can set up a mathematical bridge between the five elements of containing notes widely existent in Chinese traditional culture and abundant modern Fibonacci series related researches. The novel discovery is of great value to reduce the mysterious sense of ancient Chinese culture and is quite helpful to uncover the final scientific secret of the five periods and the six qi's theory in TCM.

\section{Materials and Methods}

2.1. Obtaining of Two Key Subsequences of Fibonacci Sequence Modulo 5. The He tu and Luo shu are two of the most famous diagrams in Chinese traditional culture as well as in TCM system. As shown in Figure 1 [1, 11], according to legend, both $\mathrm{He}$ tu and Luo shu initially emerged as groups of black and white dots, where each group of black dots has even dot number and the other groups composed of white dots have odd number of dots, respectively. In particular, either $\mathrm{He}$ tu or Luo shu has shown the same proneness to emphasize group number of 5 and the group of dots with number 5 is exactly positioned in the center of He tu and Luo shu which are arranged as the shape of a cross. Further according to the Hong fan section in Shang shu, number one is water; number two is fire; number three is wood; number four is metal; number five is soil [1]. The number of different groups of dots in He tu or Luo shu can thus form a direct association with the five elements' theory in TCM or the five elements of containing notes.

The Fibonacci sequence has a recycled property while performing modular operation by an integer $n$. While setting the value of $n$ be 5, that is, the center number of He tu or Luo 


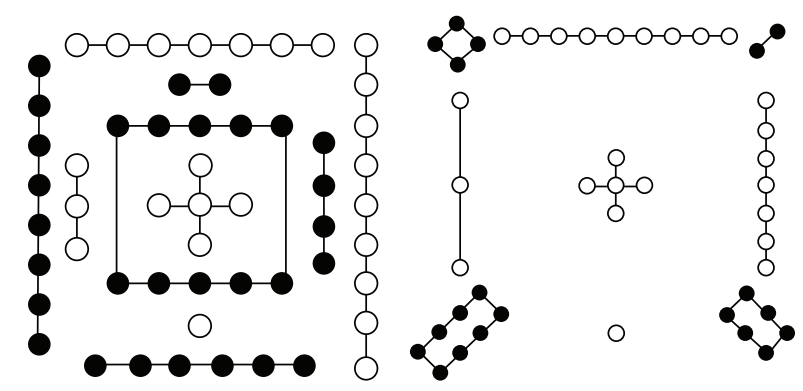

(a) The He tu diagram

(b) The Luo shu diagram

Figure 1: The He tu and Luo shu diagram.

shu, the period is equal to 20 and the corresponding recycled sequence can be listed as below [14]:

\section{0}

Suppose each index value of numbers $1,2,3,4,5,6,7$, 8,9 , and 10 in He tu or Luo shu is equal to each number itself; then no matter $\mathrm{He}$ tu and Luo shu or 10 stems and 12 branches in TCM can all be partitioned into two groups of Yin and Yang based on the parity of index values. In other words, for 10 stems and 12 branches, those with odd index numbers are attributed to the Yang group and the others with even numbers are grouped as Yin, which just corresponds to the white or black dots group in $\mathrm{He}$ tu and Luo shu, respectively. Similarly, according to parities of the index of each number the obtained 20 Fibonacci recycled sequence numbers by modulo 5 above can also be divided into two groups: subsequence Yang and subsequence Yin. The grouping result is listed as below:

\section{subsequence Yang: 1203443021 , \\ subsequence Yin: (13) 3104224013 ,}

for analyzing convenience; all the numbers in the two subsequences are rearranged into two 5-tuples and all numbers in subsequence Yin are circularly right-shifted by 2 positions. Apparently the obtained subsequences of Yang and Yin are just composed of series of 12034 and 31042 together with their corresponding palindrome, that is, two subsequences both taking 12034 and 31042 as the key sequence, respectively. In mathematics, the number 0 is just equal to 5 in the viewpoint of operation modulo 5; therefore, the key number series of 12034 and 31042 can also be seen as 12 534 and 31542 .

\subsection{New Discovery on Arrangement of the Five Elements of} Containing Notes. Based on traditional Chinese culture, each of the containing notes associates a unique symbol as well as an attribute and Hong fan number of five elements with a stem-branch combination and two neighbored stem-branch combination pairs share the same symbol and attribute [2, $3]$. The naming of the associated symbols, deriving, and relationship between such association and ancient Chinese music theory are beyond the scope of this paper; those who are interested in it can refer to related literatures directly. All stem-branch combinations and each corresponding symbol as well as the associated attribute and Hong fan number of corresponding five elements are all summarized as in Table 1 based on description in $[2,3]$.

Actually all the stem-branch combinations are just the permutation and combination between Yang group of stems and branches and Yin group of them. And the thorough building process of associations shown in Table 1 is still a mystery. While studying the rules behind the stem-branch combinations, the author found a new arrangement of the stem-branch combinations based on a simple and determinate transforming operation from the original arranging order in traditional Chinese culture which is demonstrated in Table 1 by the "number" column. The discovery of this new arrangement can help to provide research clues of mathematical structure of the five elements of containing notes. That is, take the JiaXu YiHai pair (11th and 12th stem-branch combinations in Table 1$)$ as the initial pair and take equation ( $m$ +22) $\bmod 60$ (herein "mod" represents modular operation) as a recursive rule to obtain the successive one of all recycled pairs; for example, if substituted with $m=11$ and 12, the index of the second recycled pair can be obtained; we have

$$
\begin{aligned}
& (11+22) \bmod 60=33 \\
& (12+22) \bmod 60=34 ;
\end{aligned}
$$

then look up the indexes 33 and 34 in Table 1; the second pair BingShen DingYou can be searched out. Similarly, the index of the third pair can be computed as $(33+22) \bmod 60=55$, $(34+22) \bmod 60=56, \ldots$, and finally, the whole periodical new arrangement can all be obtained. And the 5 by 6 array form of the new arrangement is shown in Table 2 .

For comparison convenience, substitute each stembranch pair in the new arrangement shown in Table 2 by its corresponding Hong fan number according to Table 1; it produces a 5 by 6 number array:

$\begin{array}{llllll}2 & 2 & 2 & 4 & 4 & 4 \\ 5 & 5 & 5 & 1 & 1 & 1 \\ 3 & 3 & 3 & 2 & 2 & 2 \\ 4 & 4 & 4 & 5 & 5 & 5 \\ 1 & 1 & 1 & 3 & 3 & 3\end{array}$

Looking up from up to bottom, it is obvious that each column of the number array is one of the recycle-shifting sequence of 12534 just by one position in the left or right 
TABLE 1: Associated stem-branch combinations, symbols, and the attributes and Hong fan numbers of five elements.

\begin{tabular}{|c|c|c|c|}
\hline Sequence number & Stem-branch combination & Symbol & Attribute and Hong fan number \\
\hline 1 & JiaZi & & Metal \\
\hline 2 & YiChou & The metal in the sea & 4 \\
\hline 3 & BingYin & & Fire \\
\hline 4 & DingMao & The fire in the stove & 2 \\
\hline 5 & WuChen & & Wood \\
\hline 6 & JiSi & The wood of a great forest & 3 \\
\hline 7 & GengWu & & Soil \\
\hline 8 & XinWei & The wayside soil & 5 \\
\hline 9 & RenShen & & Metal \\
\hline 10 & GuiYou & The metal on the sword-blade & 4 \\
\hline 11 & $\mathrm{JiaXu}$ & & Fire \\
\hline 12 & YiHai & The fire on the hill-top & 2 \\
\hline 13 & BingZi & & Water \\
\hline 14 & DingChou & The brook water & 1 \\
\hline 15 & WuYin & & Soil \\
\hline 16 & JiMao & The soil on the city-wall & 5 \\
\hline 17 & GengChen & & Metal \\
\hline 18 & $\mathrm{XinSi}$ & The metal on the white candle & 4 \\
\hline 19 & RenWu & & Wood \\
\hline 20 & GuiWei & The willow wood & 3 \\
\hline 21 & JiaShen & & Water \\
\hline 22 & YiYou & The spring water in a well & 1 \\
\hline 23 & BingXu & & Soil \\
\hline 24 & DingHai & The soil on the roof of a house & 5 \\
\hline 25 & WuZi & & Fire \\
\hline 26 & JiChou & the thundering fire & 2 \\
\hline 27 & GengYin & & Wood \\
\hline 28 & XinMao & The wood of the pine or cypress & 3 \\
\hline 29 & RenChen & & Water \\
\hline 30 & GuiSi & The flowing water & 1 \\
\hline 31 & JiaWu & & Metal \\
\hline 32 & YiWei & The metal in the sand & 4 \\
\hline 33 & BingShen & & Fire \\
\hline 34 & DingYou & The fire at the foot of a hill & 2 \\
\hline 35 & $\mathrm{WuXu}$ & & Wood \\
\hline 36 & JiHai & The wood on a plain & 3 \\
\hline 37 & GengZi & & Soil \\
\hline 38 & XinChou & The soil on the roof of a house & 5 \\
\hline 39 & RenYin & & Metal \\
\hline 40 & GuiMao & The metal on the paper money & 4 \\
\hline 41 & JiaChen & & Fire \\
\hline 42 & YiSi & The fire of a lamp under cover & 2 \\
\hline 43 & BingWu & & Water \\
\hline 44 & DingWei & The water of the heavenly river & 1 \\
\hline 45 & WuShen & & Soil \\
\hline 46 & JiYou & The soil of the highway station & 5 \\
\hline 47 & GengXu & & Metal \\
\hline 48 & XinHai & The gold of the hairpin & 4 \\
\hline 49 & RenZi & & Wood \\
\hline 50 & GuiChou & The wood of the mulberry tree & 3 \\
\hline
\end{tabular}


TABle 1: Continued.

\begin{tabular}{lccc}
\hline Sequence number & Stem-branch combination & Symbol & Attribute and Hong fan number \\
\hline 51 & JiaYin & & Water \\
52 & YiMao & The water of a great stream & 1 \\
\hline 53 & BingChen & & Soil \\
54 & DingSi & The soil in the sands & 5 \\
\hline 55 & WuWu & The heavenly fire & Fire \\
56 & JiWei & & 2 \\
\hline 57 & GengShen & The wood of the pomegranate & 3 \\
58 & XinYou & & Water \\
59 & RenXu & The water of the sea & 1 \\
\hline
\end{tabular}

TABLE 2: The 5 by 6 array form of the new arrangement.

\begin{tabular}{lccccc}
\hline JiaXu YiHai & BingShen DingYou & WuWu JiWei & GengChen XinSi & RenYin GuiMao & JiaZi YiChou \\
BingXu DingHai & WuShen JiYou & GengWu XinWei & RenChen GuiSi & JiaYin YiMao & BingZi DingChou \\
WuXu JiHai & GengShen XinYou & RenWu GuiWei & JiaChen YiSi & BingYin DingMao & WuZi JiChou \\
GengXu XinHai & RenShen GuiYou & JiaWu YiWei & BingChen DingSi & WuYin JiMao & GengZi XinChou \\
RenXu GuiHai & JiaShen YiYou & BingWu DingWei & WuChen JiSi & GengYin XinMao & RenZi GuiChou \\
\hline
\end{tabular}

TABLE 3: New arrangement based on the 12 branches.

\begin{tabular}{lccccc}
\hline JiaZi YiChou4 & BingYin DingMao2 & WuChen JiSi3 & GengWu XinWei5 & RenShen GuiYou4 & JiaXu YiHai2 \\
BingZi DingChou1 & WuYin JiMao5 & GengChen XinSi4 & RenWu GuiWei3 & JiaShen YiYoul & BingXu DingHai5 \\
WuZi JiChou2 & GengYin XinMao3 & RenChen GuiSi1 & JiaWu YiWei4 & BingShen DingYou2 & WuXu JiHai3 \\
GengZi XinChou5 & RenYin GuiMao4 & JiaChen YiSi2 & BingWu DingWeil & WuShen JiYou5 & GengXu XinHai4 \\
RenZi GuiChou3 & JiaYin YiMao1 & BingChen DingSi5 & WuWu JiWei2 & GengShen XinYou3 & RenXu GuiHail \\
\hline
\end{tabular}

direction, that is, 25341 or 41253 , and looking from the end there are exactly three appositions of 3154231542.

Above discovery is not only a special case. Stem-branch combinations and their corresponding Hong fan number given in Table 1 can also derive two other arrangements as listed in Tables 3 and 4 based on the 12 branches or base number 8 after extending one duplicate of whole 60 elements.

Obviously, in each column of Table 3 the Hong fan numbers are the recycle-shifting sequence of 12534 by a certain number. And in each column of Table 4, by recycling view, the Hong fan numbers all show a similar appositional pattern of three same recycle-shifting sequences of 31542 .

\section{Results, Discussions, and Conclusions}

In this paper, enlightened by elementary Yin or Yang number grouping principle of $\mathrm{He}$ tu, Luo shu, and stem-branch theory, the 12534 and 31542 key number series of Fibonacci sequence modulo 5 (the center number of $\mathrm{He}$ tu or Luo shu) are obtained. And by simple and determinate transforming operation of original order of the stem-branch combinations widely existent in traditional Chinese culture, a new 5 by 6 arrangement about the five elements of containing notes is firstly introduced, which has shown close relationship with the two obtained key subsequences of Fibonacci sequence modulo 5. As the new derived arrangement is directly acquired from the original indices of stem-branch combinations by fixed mathematical operation, the rule implied in the new arrangement also reflects that of the original arrangement of stems and branches in traditional Chinese culture. Another two derived arrangements about the five elements of containing notes also show close relationship with sequence 12534 or 31542 respectively; therefore it can be deduced that the five elements of containing notes must have underlying profound mathematical relationship with the Fibonacci modular sequence. Actually, besides applications in fate judgments or in ancient Taoist system, the five elements of containing notes associated with 60 stem-branch combinations have also close relationship with traditional Chinese musical system [3]. And for ancient Chinese people, it is very common and natural to describe the musical and calendric laws in the same form based on stems and branches. In their opinions, the climate throughout a whole year is closely related with certain musical notes denoted by a stembranch combination corresponding to the year as the five periods and six qi in TCM do. Moreover, the Fibonacci sequence widely exists in various modern disciplines especially in the biological world, and as a special traditional medicine system, herbs from the biological world are widely used in TCM. Therefore, although researches in this paper are mainly performed based on arrangements of the five elements of containing notes, considering it almost shares a 
TABLE 4: New arrangement based on base number 8 after extending one duplicate of 60 elements.

\begin{tabular}{lccc}
\hline Jiazi YiChou4 & BingYin DingMao2 & WuChen JiSi3 & GengWu XinWei5 \\
RenShen GuiYou4 & Jiaxu YiHai2 & WingZi DingChoul & BingXu DingHai5 \\
GengChen XinSi4 & RenWu GuiWei3 & JiaShen YiYoul & JiaWu YiWei4 \\
WuZi JiChou2 & GengYin XinMao3 & RenChen GuiSi1 & RenYin GuiMao4 \\
BingShen DingYou2 & WuXu JiHai3 & GengZi XinChou5 & GengXu XinHai4 \\
JiaChen YiSi2 & BingWu DingWeil & WuShen JiYou5 & WuWu JiWei2 \\
RenZi GuiChou3 & JiaYin YiMao1 & BingChen DingSi5 & BingYin DingMao2 \\
GengShen XinYou3 & RenXu GuiHai1 & JiaZi YiChou4 & JiaXu YiHai2 \\
WuChen JiSi3 & GengWu XinWei5 & RenShen GuiYou4 & RenWu GuiWei3 \\
BingZi DingChou1 & WuYin JiMao5 & GengChen XinSi4 & GengYin XinMao3 \\
JiaShen YiYou1 & BingXu DingHai5 & WuZi JiChou2 & WuXu JiHai3 \\
RenChen GuiSi1 & JiaWu YiWei4 & BingShen DingYou2 & BingWu DingWei1 \\
GengZi XinChou5 & RenYin GuiMao4 & JiaChen YiSi2 & JiaYin YiMao1 \\
WuShen JiYou5 & GengXu XinHai4 & RenZi GuiChou3 & RenXu GuiHail \\
BingChen DingSi5 & WuWu JiWei2 & GengShen XinYou3 &
\end{tabular}

common basis of stem-branch system with the theory of the five periods and six qi in TCM, the mathematical relationship of five elements of containing notes and Fibonacci sequence modulo 5 found in this paper is undoubtedly quite helpful to recover the scientific secret of the five periods and six qi's theory in TCM as well as that of whole traditional Chinese culture system, but more data is still needed to elucidate the TCM related theory in future researches.

\section{Conflict of Interests}

The author declares that there is no conflict of interests regarding the publication of this paper.

\section{Authors' Contributions}

Zhaoxue Chen carried out all the studies of this paper and drafted the paper, as well as reading and approving the final paper.

\section{Acknowledgments}

This work is under the auspice of Shanghai Municipal Education Commission to Scientific Innovation Research Funds (no. 11YZ116) and BME first-class discipline (B) building project for Shanghai universities.

\section{References}

[1] P. Unschuld, Huang Di Nei Jing Su Wen: Nature, Knowledge, Imagery, in an Ancient Chinese Medical Text, University of California Press, Berkeley, Calif, USA, 2003.

[2] W.-P. Chao and Z. W. Bang, "The Chinese science of fatecalculation," Folklore Studies, vol. 5, pp. 279-315, 1946.

[3] H. Datong, “On '60 jiazi containing notes' study," Studies in Culture \& Art, no. 4, pp. 64-98, 2009.

[4] Y. Tu, "The discovery of artemisinin (qinghaosu) and gifts from Chinese medicine," Nature Medicine, vol. 17, no. 10, pp. 12171220, 2011.
[5] C. Zhou, Fibonacci-Lucas Sequences and Their Applications, Hunan Science and Technology Publishing House, Changsha, China, 1993.

[6] Z.-X. Chen, Z.-Z. Wang, Y. Sun, and F.-L. Bei, "Discussion on microcosmic derivation of biological golden section phenomena from DNA geometric structure and snow flower generation," Interdisciplinary Sciences: Computational Life Sciences, vol. 3, no. 1, pp. 31-35, 2011.

[7] V. Weiss and H. Weiss, "The golden mean as clock cycle of brain waves," Chaos, Solitons and Fractals, vol. 18, no. 4, pp. 643-652, 2003.

[8] C. J. Glasby, S. P. Glasby, and F. Pleijel, "Worms by number," Proceedings of the Royal Society B: Biological Sciences, vol. 275, no. 1647, pp. 2071-2076, 2008.

[9] C. R. Li, X. N. Zhang, and Z. X. Cao, "Triangular and Fibonacci number patterns driven by stress on core/shell microstructures," Science, vol. 309, no. 5736, pp. 909-911, 2005.

[10] http://www.fq.math.ca/.

[11] E. Moran, M. V. Biktashev, V. Biktashev, and J. Yu, The Complete Idiot's Guide to Feng Shui, Pearson Education, 2nd edition, 2002.

[12] Z. Gao, Quest on Secret of Jiugong Diagram, Hongkong Pegasus Books Limited Company, Hong Kong, 2004.

[13] J. Geng, "Relationship between Loshu and the Fibonacci sequence," Bulletin of Mathematics, vol. 47, no. 5, pp. 46-49, 2008.

[14] M. Renault, Properties of the Fibonacci sequence under various moduli [M. S. thesis], Wake Forest University, Winston-Salem, Calif, USA, 1996. 

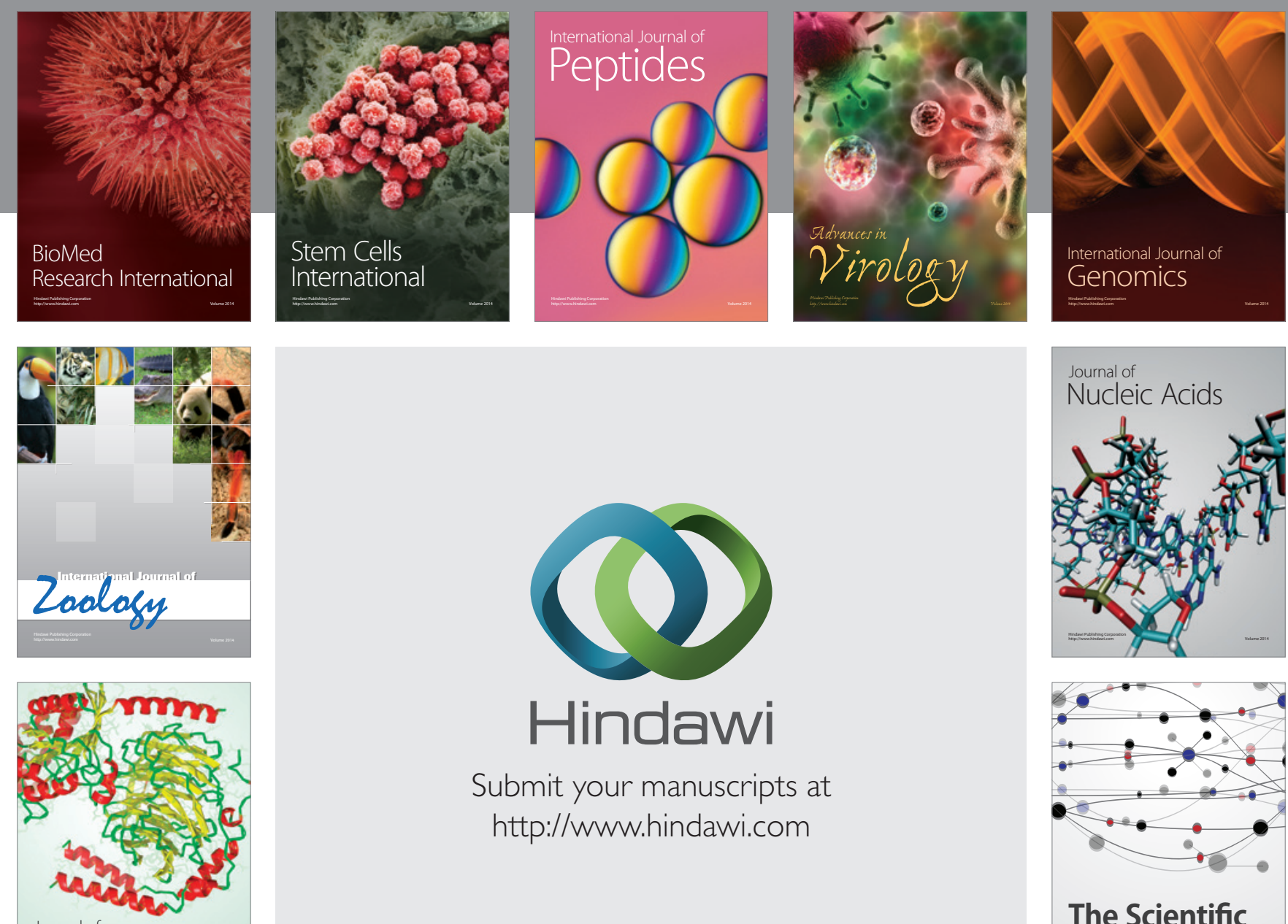

Submit your manuscripts at

http://www.hindawi.com

Journal of
Signal Transduction
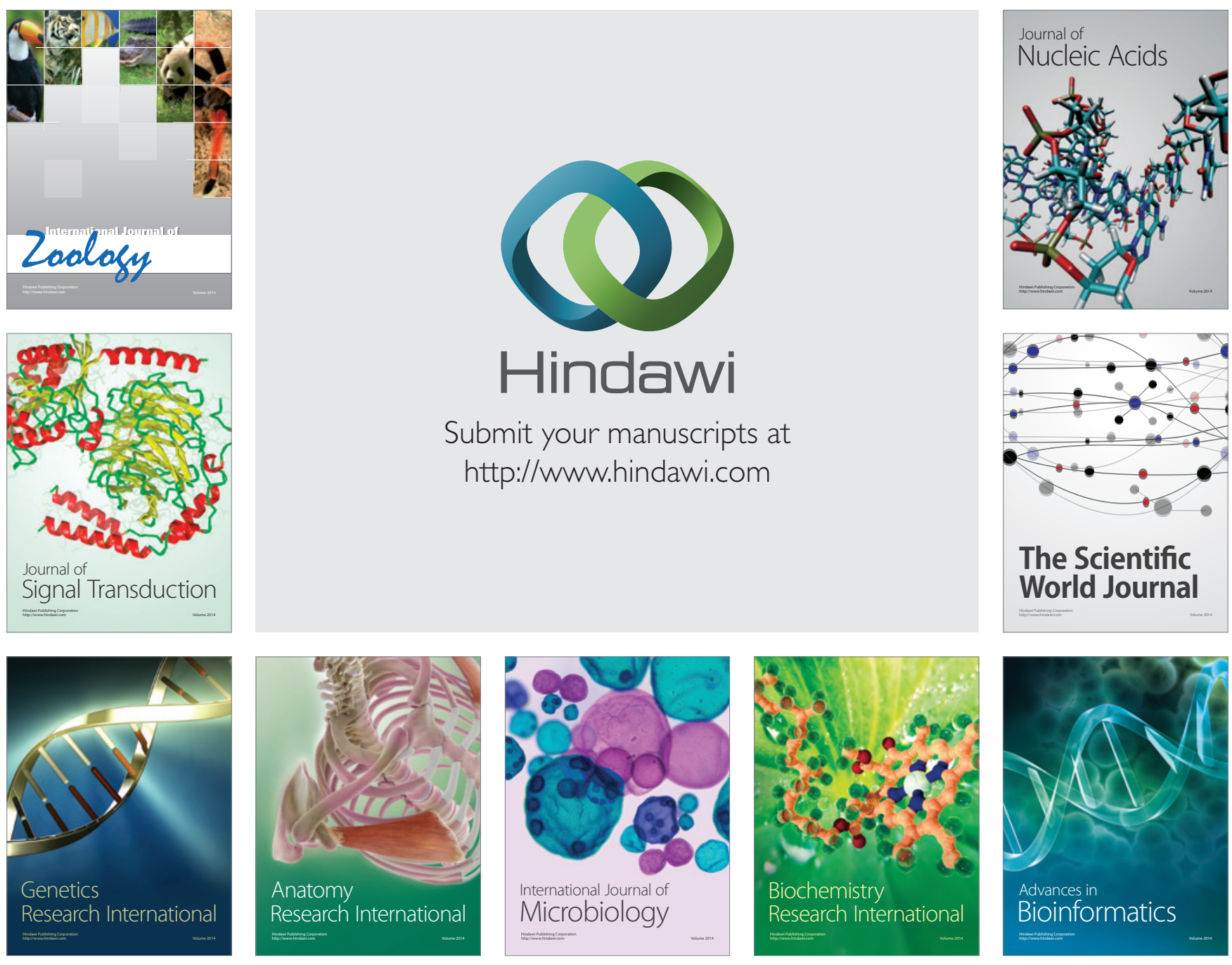

The Scientific World Journal
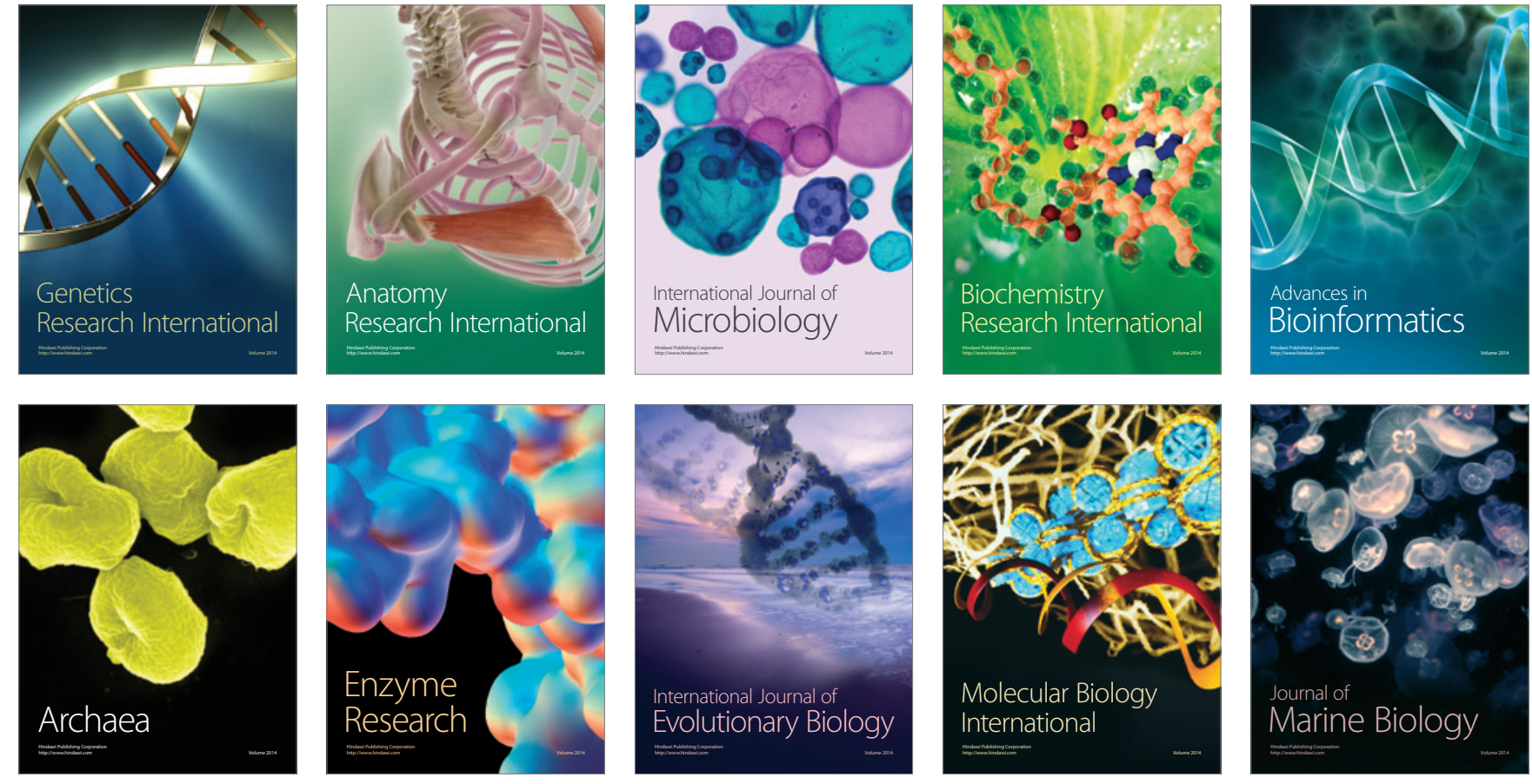